\title{
Theoretical and Experimental Investigation of the Nonlinear Behavior of an Electrostatically Actuated In-plane MEMS Arch
}

\author{
Abdallah H Ramini, Qais M Hennawi, and Mohammad I Younis, Member, ASME
}

\begin{abstract}
We present theoretical and experimental investigation of the nonlinear behavior of a clamped-clamped in-plane MEMS arch when excited by a DC electrostatic load superimposed to an AC harmonic load. Experimentally, a case study of in-plane silicon micromachined arch is examined and its mechanical behavior is measured using optical techniques. An algorithm is developed to extract the various parameters, such as the induced axial force and the initial rise, needed to model the behavior of the arch. A softening spring behavior is observed when the excitation is close to the first resonance frequency due to the quadratic nonlinearity coming from the arch geometry and the electrostatic force. Also, a hardening spring behavior is observed when the excitation is close to the third (second symmetric) resonance frequency due to the cubic nonlinearity coming from mid-plane stretching. Dynamic snap-through behavior is also reported for larger range of electric loads. Theoretically, a multimode Galerkin reduced order model is utilized to simulate the arch behavior. General agreement is reported among the theoretical and experimental data.
\end{abstract}

Index Terms-Arch, resonator, parameter extraction, pull-in instability, nonlinear dynamics, snap-through instability.

\section{INTRODUCTION}

$\mathrm{M}$ ICROELECTOMECHANICAL arches have gained significant interests in the recent years because of their desirable static and dynamic characteristics for many applications. These structures have two stable states to hold on if they are used for static applications, thus saving actuation power. The bistability nature of arches gives them the advantage of exhibiting large motion stroke when moving between the two stable states, which can be advantageous for resonators and other actuation applications because it yields significantly large signal-to-noise ratio. Consequently, the bistability of arches has been thoroughly studied in order to exploit them in useful applications [1-10]. For example, micro arches are used as microbridges [7], microvalves [11], microelectro switches or relays [12], logic memories [13], micromuscles [14], and micro-optical switches [15].

Manuscript received XXXXXX XX, 20XX.

This work was supported by the King Abdullah University of Science and Technology, Thuwal, Saudi Arabia. The authors are with the King Abdullah University of Science and Technology, Thuwal 23955-6900, Saudi Arabia (corresponding author Mohammad Younis,

Mohammad.Younis@kaust.edu.sa).
Micro arches were excited using various transduction methods including magnetic [6], electrostatic [10], and thermal methods [12]. However, electrostatic sensing and actuation remains the most attractive method because of its distinguished features, such as the fast speed, ease of fabrication, high actuating force, and large controllability [16]. Also, the interaction between the nonlinearities involved in the arch with the inherently nonlinear electrostatic force results in interesting nonlinear phenomena, such as snap-through, pullin, hysteresis, softening or hardening behaviors, co-existence of states, and secondary resonances.

The mechanical behaviors of an arch, particularly its snapthrough and its pull-in instabilities have been studied considerably to understand their static and dynamic features. Das et al. [2] studied the snap-through and pull-in instabilities of parabolic- and bell-shaped clamped-clamped micro arches. Zhang et al. [3] derived an analytical expression to predict the snap-through and pull-in instabilities. Krylov et al. [4] studied the effect of the arch parameters like the thickness and the initial rise on the relative location of the snap-through and pull-in voltages. They came up with a closed form formula to compute the critical initial rise, which guarantees the existence of the snap-through instability. Sari and Pakdemirli [17] studied slightly curved resonant microbeam having non-ideal boundary conditions resting on elastic foundation. Plaut [18] studied the snap-through instability of arches and buckled beams and computed the critical bucking displacements for different beams under different boundary conditions. Hu et al. [19] investigated exploiting the buckling phenomenon in arches and buckled beams in smart systems.

Many people studied the natural frequencies and mode shapes of arches. Nayfeh et al. [20] derived the eigenvalue problem of buckled beams with different supporting conditions including hinged-hinged, clamped-clamped, and hingedclamped conditions. They validated the theoretical results with experiment, reporting excellent agreement. Dawe [21] calculated the natural frequencies of a shallow arch using the discrete element displacement method neglecting the longitudinal inertia. For micro scale arches, Ouakad and Younis [22] solved the eigenvalue problem for an arch considering the effect of the DC voltage load, and then they demonstrated the effect of changing the initial rise and the DC voltage load on the natural frequencies and mode shapes. Alkharabsheh and Younis [23] studied the effect of the axial force on the fundamental natural frequency of the arch for various values of the DC voltage loads. It was found that applying a tensile axial force has a softening effect, and hence reducing the natural frequency. On the other hand, applying a compressive load has a hardening behavior, and hence increasing the natural frequency. 
Mallona et al. [24] investigated the quasi-static and nonlinear dynamic behavior of a shallow arch subjected to a dynamic pulse load. They revealed that the critical shock load can be varied by controlling the shape of the arch. Lv et al. [25] studied the effect of geometrical imperfections on the dynamic stability of a hinged-hinged sinusoidal shallow arch subjected to a time varying distributed load. They compared the results with similar study carried on a perfect arch to find the effect of imperfections. Ruzziconi et al. [26] investigated theoretically and experimentally the response of an electrostatically actuated imperfect microbeam. They found experimentally the mode shapes associated with the arch. They performed several frequency sweeps forward and backward near the first modal frequency. They developed a two-degree-of-freedom reduced order model based on the Galerkin approach. Buchaillot et al. [27] studied the dynamic snap-through phenomenon on highly-raised buckled micro beams. They fabricated and tested micro clamped-clamped buckled beams with high vibration amplitudes. Because of the high initial rise, there was an inversion between the first two natural frequencies. Alkharabsheh and Younis [28] studied the dynamics of an electrostatically-actuated shallow arch including the effect of flexible supports at the boundaries. They modeled the boundary conditions by both translational and rotational springs. They used the shooting method to solve for the periodic solutions and assessed the stability of the found solutions using the Floquet theory. Medina et al. [29] studied experimentally the stability of micro beams subjected to an induced axial force and electrostatic loading. They used the deviation of the arch rise from the designed value to get the value of the induced axial force. Younis et al. [30] investigated the nonlinear statics and dynamics of an electrostatically actuated MEMS clamped-clamped arch. They utilized a multi-mode reduced order model and studied the dynamics of the arch near its fundamental and third resonance frequencies as well as near subharmonic resonances. Medina et al [32] showed experimentally on an arched microbeam a transition from the initial buckled position to the other inaccessible buckling position and trapped it at that buckled position using two step electrostatic loading.

This paper focuses on clamped-clamped bulk micromachined shallow arches. Reviewing the previous works on electrostatically actuated micro arches, one can notice that there are several studies about the static and dynamic behaviors of arches. However, the majority of the works focused on the static and transient behaviors with little attention on the nonlinear dynamic behavior, especially due to harmonic excitations. No experimental investigation was presented showing dynamic snap-through at the micro scale. In this paper, we present an experimental and theoretical investigation for the dynamic behavior of in-plane arches. Through this study, we show dynamic snap-through behaviors, co-existence, and hardening and softening behaviors. These nonlinear phenomena can be exploited for applications in the field of sensing and actuation.

The organization of this paper is as follows; Section II formulates the problem and derives the reduced order model mathematically. Section III presents the arch design and the characterization details. Section IV introduces the method used to extract the model parameters. Section V shows the experimental data and comparison between experimental data with the theoretical model. Finally, we conclude the paper in Section VI.

\section{PROBLEM FORMULATION}

We consider an initially curved clamped-clamped arch of effective length $L$, width $b$, thickness $h$, mass density $\rho$, and initial profile,

$$
\hat{w}_{o}(\hat{x})=-\frac{b_{o}}{2}\left(1-\cos \left(\frac{2 \pi \hat{x}}{L}\right)\right)
$$

where $\hat{x}$ is the position along the horizontal direction of the arch and $b_{o}$ is the mid-span initial rise. The arch is actuated electrostatically using a DC voltage load of amplitude $V_{D C}$ superimposed to an AC harmonic voltage load of amplitude $V_{A C}$ and frequency $\hat{\Omega}$ by an electrode with a separation gap $d$. The transverse deflection $\hat{w}(\hat{x}, \hat{t})$ in time $\hat{t}$ assuming an EulerBernoulli model and a small initial slope $\hat{w}_{o}^{\prime}(\hat{x}) \ll<$ can be written [22] as

$$
\begin{aligned}
& E I \frac{\partial^{4} \hat{w}}{\partial \hat{x}^{4}}+\rho A \frac{\partial^{2} \hat{w}}{\partial \hat{t}^{2}}+\hat{c} \frac{\partial \hat{w}}{\partial \hat{t}}=\frac{\varepsilon b\left[V_{D C}+V_{A C} \cos (\hat{\Omega} \hat{t})\right]^{2}}{2\left(d-\hat{w}_{0}-\hat{w}\right)^{2}} \\
& +\left[\frac{\partial^{2} \hat{w}}{\partial \hat{x}^{2}}+\frac{d^{2} \hat{w}_{0}}{d \hat{x}^{2}}\right]\left[\hat{N}+\frac{E A}{2 L} \int_{0}^{L}\left\{\left(\frac{\partial \hat{w}}{\partial \hat{x}}\right)^{2}+2\left(\frac{\partial \hat{w}}{\partial \hat{x}}\right)\left(\frac{d \hat{w}_{0}}{d \hat{x}}\right)\right\} d \hat{x}\right]
\end{aligned}
$$

where $A=h b$ is the cross section area, $I=b h^{3} / 12$ is the area moment of inertia, $\hat{N}$ is the induced internal axial force, and $E$ is the effective Young's modulus of the arch material. Since the width is very large compared to the thickness $b>5 h$, we use the cylindrical plate theory assumption $E_{\text {effective }}=E /\left(1-v^{2}\right)$, where $v$ is Poisson's ratio.

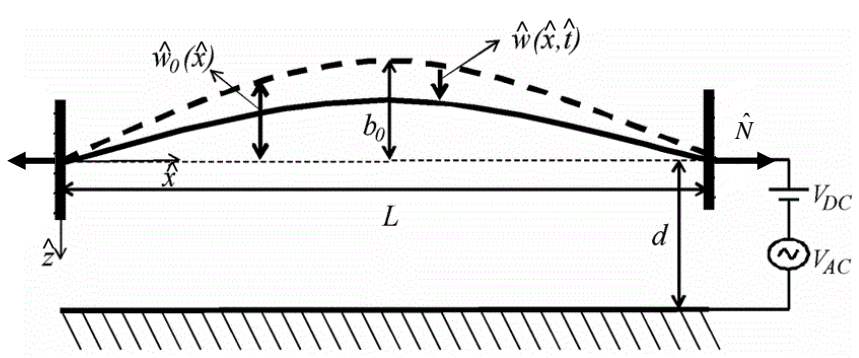

Fig.1. Schematic diagram of the micro arch.

The boundary conditions are assumed to be perfectly clamped at both ends such that

$$
\begin{array}{ll}
\hat{w}(0, \hat{t})=0 ; & \frac{\partial \hat{w}}{\partial \hat{x}}(0, \hat{t})=0 \\
\hat{w}(L, \hat{t})=0 ; & \frac{\partial \hat{w}}{\partial \hat{x}}(L, \hat{t})=0
\end{array}
$$

For convenience, the following nondimensional variables are introduced: 


$$
w=\frac{\hat{w}}{d} ; \quad w_{0}=\frac{\hat{w}_{0}}{d} ; \quad x=\frac{\hat{x}}{L} ; \quad t=\frac{\hat{t}}{T}
$$

where $T$ is a time scale constant chosen as $T=\sqrt{\rho A L^{4} / E I}$. Therefore, the nondimensional equation becomes

$$
\begin{aligned}
& \frac{\partial^{4} w}{\partial x^{4}}+\frac{\partial^{2} w}{\partial t^{2}}+c \frac{\partial w}{\partial t}=\alpha_{2} \frac{\left(V_{D C}+V_{D C} \operatorname{Cos}(\Omega t)\right)^{2}}{2\left(1-w_{o}-w\right)^{2}} \\
& +\left(\frac{\partial^{2} w}{\partial x^{2}}+\frac{d^{2} w_{o}}{d x^{2}}\right)\left(\alpha_{1} \int_{0}^{1}\left\{\left(\frac{\partial w}{\partial x}\right)^{2}+2\left(\frac{\partial w}{\partial x} \frac{\partial w_{o}}{\partial x}\right)\right\} d x+N\right)
\end{aligned}
$$

The nondimensional boundary conditions are

$$
\begin{aligned}
w(0, t) & =0 ; & & \frac{\partial w}{\partial x}(0, t)=0 \\
w(1, t) & =0 ; & \frac{\partial w}{\partial x}(1, t) & =0
\end{aligned}
$$

and also, the parameters of equation (5) are defined as

$$
\begin{array}{cl}
\alpha_{1}=6\left(\frac{d}{h}\right)^{2} ; & \alpha_{2}=\frac{\varepsilon b L^{4}}{2 E I d^{3}} ; \quad \Omega=\frac{\hat{\Omega}}{\omega_{n}} ; \quad \omega_{n}=\frac{1}{T} \\
c=\frac{\hat{c} L^{4}}{E I T} ; & N=\frac{\hat{N} L^{2}}{E I} ; \quad w_{o}(x)=-\frac{b_{o}}{2 d}[1-\cos (2 \pi x)]
\end{array}
$$

Equation (5) is a nonlinear integro-partial differential equation with no available analytical solution. Therefore, approximate methods need to be used. Here we employ the Galerkin approach to derive a Reduced Order Model (ROM) [16]. Thus, the transverse deflection is assumed

$$
w(x, t)=\sum_{i=1}^{n} \varphi_{i}(x) u_{i}(t)
$$

where, $\varphi_{i}(x)$ is the normalized linear undamped $i^{\text {th }}$ mode shape of a straight clamped-clamped beam given by

$$
\varphi_{i}(x)=\cos \left(\sigma_{i} x\right)-\cosh \left(\sigma_{i} x\right)-\psi_{i}\left(\sin \left(\sigma_{i} x\right)-\sinh \left(\sigma_{i} x\right)\right)
$$

where,

$$
\begin{aligned}
& \sigma_{i}=\frac{2 i+1}{2}, \quad i=1,2,3, \ldots \\
& \psi_{i}=\frac{\cos \left(\sigma_{i} L\right)-\cosh \left(\sigma_{i} L\right)}{\sin \left(\sigma_{i} L\right)-\sinh \left(\sigma_{i} L\right)}, \quad i=1,2,3, \ldots
\end{aligned}
$$

$u_{i}(t)$ is the time varying modal coordinate function corresponding to the mode shape $\varphi_{i}(x)$.

Next, equation (5) is multiplied by the denominator of the electrostatic force term $\left(1-w_{o}-w\right)^{2}$ in order to reduce the computational cost $[31,33]$. Then, substituting equation (8) into the resulting equation, multiplying by the mode shape $\varphi_{j}(x)$, and then integrating the outcome over the normalized domain $x \in[0,1]$ yields

$$
\begin{gathered}
\sum_{i=1}^{n}\left(M_{i j} \ddot{u}_{i}(t)+C_{i j} \dot{u}_{i}(t)+K_{i j} u_{i}(t)\right)= \\
\alpha_{1} \Lambda_{i}\left\{\left(\sum_{i=1}^{n} \varphi_{i}^{\prime}(x) u_{i}(t)\right)^{2}+2 w_{0}^{\prime}\left(\sum_{i=1}^{n} \varphi_{i}^{\prime}(x) u_{i}(t)\right)\right\} d x \\
+\alpha_{2} V^{2}(t) \int_{0}^{1} \varphi_{j}(x) d x \\
i=1,2,3, \ldots, n
\end{gathered}
$$

where,

$$
\begin{aligned}
& M_{i j}=\int_{0}^{1} \varphi_{j}(x) \varphi_{i}(x)\left[1-w_{0}-\sum_{i=1}^{n} \varphi_{i}(x) u_{i}(t)\right]^{2} d x \\
& C_{i j}=c \int_{0}^{1} \varphi_{j}(x) \varphi_{i}(x)\left[1-w_{0}-\sum_{i=1}^{n} \varphi_{i}(x) u_{i}(t)\right]^{2} d x \\
& K_{i j}=\int_{0}^{1} \varphi_{j}(x) \varphi_{i}^{(4)}(x)\left[1-w_{0}-\sum_{i=1}^{n} \varphi_{i}(x) u_{i}(t)\right]^{2} d x
\end{aligned}
$$

and,

$$
\begin{aligned}
& \Lambda_{i}=\int_{0}^{1} \varphi_{j}(x)\left\{\left[1-w_{0}-\sum_{i=1}^{n} \varphi_{i}(x) u_{i}(t)\right]^{2}\left[w_{0}^{\prime \prime}(x)+\sum_{i=1}^{n} \varphi_{i}^{\prime \prime}(x) u_{i}(t)\right] d x\right\} ; \\
& V(t)=V_{D C}+V_{A C} \cos (\Omega t) .
\end{aligned}
$$

\section{DESIGN AND CHARACTERIZATION}

The MEMS arch under study was designed as initially curved clamped microbeam, which forms one side of a parallel plate capacitor. The arch is designed to move in the plane of the chip because of the flexibility to move with a large stroke. Fig. 2 shows a schematic diagram of the in-plane arch.

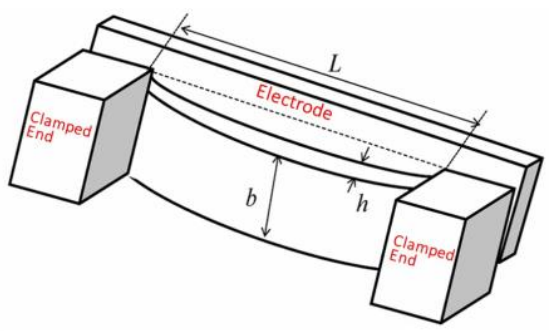

Fig.2. Schematic view of the in-plane arch.

The investigated MEMS arch was fabricated by MEMSCAP [34] from SOI wafers with highly conductive $25 \mu \mathrm{m}$ Si device layer through Deep Reactive Ion Etching (DRIE) to pattern the gaps between the structure and the electrodes. The cross section of the arch is assumed to be rectangular cross section with uniform thickness $h$ throughout the length $L$ and the width $b$. The profile of the arch is characterized optically using an interferometry profilometer. Fig. 3 shows an SEM image of the tested arch. It should be pointed that although this figure shows the arch sandwiched between two electrodes; only one electrode is activated in this work. 


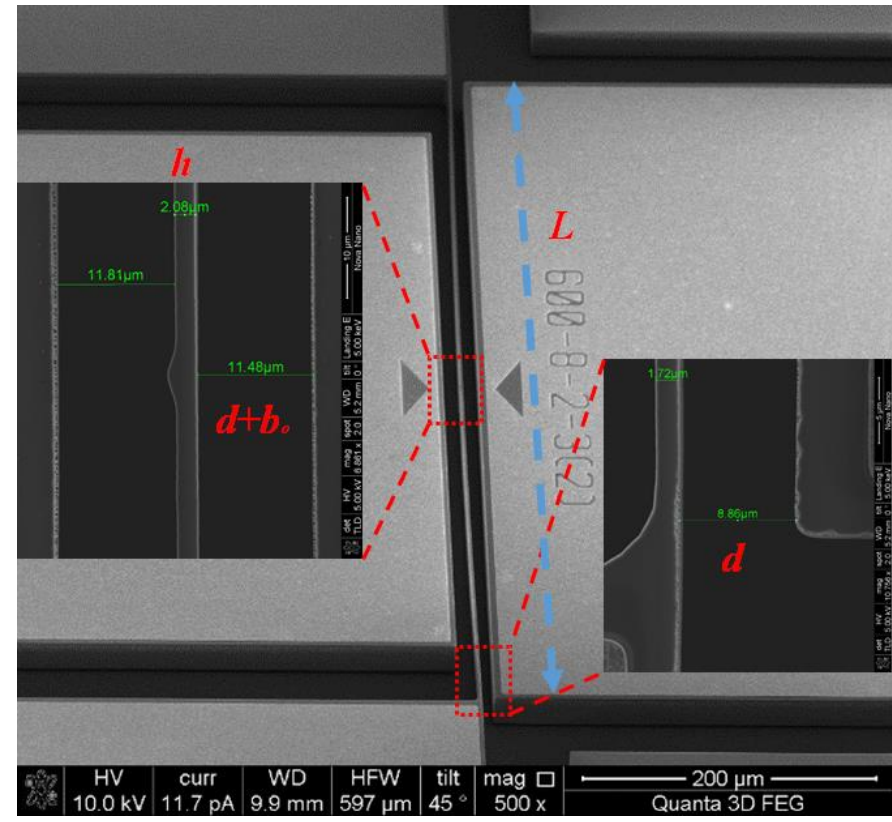

Fig.3. SEM images of the in-plane arch.

\section{PARAMETERS EXTRACTION METHODOLOGY}

Due to the axial stress induced by the fabrication process, as well as other imperfections, the initial intended profile of the micro arch differs from the actual measured one $w_{s}$. This ultimately affects the resonance frequencies and the dynamic behavior of the arch. Consequently, the unknown parameters to be extracted to be able to model the arch are the induced axial force $N$ due to the fabrication, the original initial rise before this induced axial stresses $b_{o}$ and hence $w_{o}$ in equation (1), in addition to the Young's modules E. The known parameters, which are measured experimentally are the initial rise after the etching process $b_{s}$, and hence the whole profile $w_{s}$ is known, and the linear first and third (second symmetric) resonance frequencies of the $\operatorname{arch}, f_{1}$ and $f_{3}$, respectively, obtained from ring down measurements that is independent of the electrostatic voltage (details in Section V). It should be mentioned that the thickness of the arch beam $h$ varies slightly along the width and the length of the arch beam. But in this paper, we consider it to be constant throughout our numerical simulations and is taken equal $h=2 \mu \mathrm{m}$.

Next, we describe the equations that govern the above known and unknown parameters of the arch.

We start by deriving the static equation governing the new profile of the $\operatorname{arch} w_{s}$ due to the axial load $N$. For this, we drop the damping, time varying, and electrostatic forcing terms in equation (5), which yields

$$
\begin{aligned}
& \frac{d^{4} w_{s}}{d x^{4}}= \\
& \left(\frac{d^{2} w_{s}}{d x^{2}}+\frac{d^{2} w_{o}}{d x^{2}}\right)\left(\alpha_{1} \int_{0}^{1}\left\{\left(\frac{d w_{s}}{d x}\right)^{2}+2\left(\frac{d w_{s}}{d x} \frac{d w_{o}}{d x}\right)\right\} d x+N\right)
\end{aligned}
$$

To obtain the equation governing the natural frequencies, we linearize equation (5) around $w_{s}$ and drop the damping and electrostatic forcing terms. Toward this, we assume

$$
w(x, t)=w_{s}+V(x, t)
$$

where $V(x, t)$ is the arch deflection with respect to $w_{s}$. Substituting equation (15), dropping the equilibrium equation, equation (14), and retaining the linear terms in equation (5) gives

$$
\begin{aligned}
& \frac{\partial^{4} V}{\partial x^{4}}+\frac{\partial^{2} V}{\partial t^{2}}= \\
& \frac{\partial^{2} V}{\partial x^{2}}\left(\alpha_{1} \int_{0}^{1}\left\{\left(\frac{d w_{s}}{d x}\right)^{2}+2 \frac{d w_{s}}{d x} \frac{d w_{o}}{d x}\right\} d x+N\right) \\
& +\left(\frac{d^{2} w_{s}}{d x^{2}}+\frac{d^{2} w_{o}}{d x^{2}}\right) \alpha_{1} \int_{0}^{1}\left\{2 \frac{\partial V}{\partial x}\left(\frac{d w_{s}}{d x}+\frac{d w_{o}}{d x}\right)\right\} d x
\end{aligned}
$$

To derive the eigenvalue problem, we use separation of variables in equation (16) and assume

$$
V(x, t)=\phi_{n}(x) e^{i \omega_{n} t}
$$

The outcome equation is

$$
\begin{aligned}
& \frac{d^{4} \phi_{n}}{d x^{4}}-\omega_{n}^{2} \phi_{n}= \\
& \frac{d^{2} \phi_{n}}{d x^{2}}\left(\alpha_{1} \int_{0}^{1}\left\{\left(\frac{d w_{s}}{d x}\right)^{2}+2 \frac{d w_{s}}{d x} \frac{d w_{o}}{d x}\right\} d x+N\right) \\
& +\left(\frac{d^{2} w_{s}}{d x^{2}}+\frac{d^{2} w_{o}}{d x^{2}}\right) \alpha_{1} \int_{0}^{1}\left\{2 \frac{d \phi_{n}}{d x}\left(\frac{d w_{s}}{d x}+\frac{d w_{o}}{d x}\right)\right\} d x
\end{aligned}
$$

We solve the above eigenvalue problem using the Galerkin approach [16]. This yields two equations governing the first and third natural frequencies. Solving these with the third equation, equation (14), yields the three unknowns.

The numerical procedure is as follows. We assume an initial guess for the initial rise $b_{o}$, and hence $w_{o}$, and also iterate on the nondimensional axial force $N$, then solve the eigen value problem of equation (18) to match the ratio of the nondimensional frequencies $\omega_{3} / \omega_{1}$ with the ratio of the experimentally measured resonance frequencies $f_{3} / f_{l}$. It is worth mentioning that the measured resonance frequencies are corrected to account for the measured damping ratios of each mode.

Then, we solve the static deflection $w_{s}$, and hence $b_{s}$, from equation (14) due to the assumed $N$ and $b_{o}$ and compare with the measured value. If the calculated $b_{s}$ matches the experimentally measured value (in our case $b_{s}=2.6 \mu \mathrm{m}$ ), then our assumptions of $b_{o}$ and $N$ are correct. Otherwise, $b_{o}$ and $N$ are updated until achieving convergence. After convergence, we calculate the Young's modulus $E$ using

$$
E=\frac{4 \pi^{2} b h L^{4}}{I} \frac{f_{1}^{2}}{\omega_{1}^{2}}
$$

Finally, we calculate the dimensional axial force by recalling equation (7) and the relationship between $N$ and $\hat{N}$. Fig. 4 shows schematic of the applied algorithm. 
In this work, the measured parameters of the arch considered are shown in Table I. The experimentally obtained frequencies are $f_{1}=86.6 \mathrm{kHz}$ for the first symmetric resonance frequency and $f_{3}=247 \mathrm{kHz}$ for the third resonance frequency. After iterating the algorithm, the ratio between the frequencies match at the value of the initial rise $b_{o}=2.95 \mu \mathrm{m}$. Also, this ratio corresponds to a nondimensional tension axial force $N$ $=19$. Using equations (17) and (18), the Young's modulus $E$ equals $110 \mathrm{GPa}$ and the dimensional tensile axial force $\hat{N}$ equals $0.097 \mathrm{mN}$.

It is noted above that the extracted value of $E$ is below the range reported in the literature for silicon [35]. Since $E$ is always associated with $I$ (the moment of inertia of the cross section), and $A$ (the area of the cross section), as clear in Eq. (2), and since we have experimental evidences of the nonuniformity of both thickness $h$ and beam width $W$, it seems that this low value reflects an error in the assumption of constant $h$ and $W$. To correct this; one needs to seek accurate description of the geometry as it varies along the beam length, by measurements and enter this into the equation of motion, and run the parameters extraction algorithm again.

TABLE I. Geometrical properties of the tested arch.

\begin{tabular}{lc}
\hline Parameter & Value $(\mu \mathrm{m})$ \\
\hline Length $(L)$ & 600 \\
Width $(b)$ & 25 \\
Rise $\left(b_{s}\right)$ & 2.6 \\
Gap $(d)$ & 8.86 \\
Thickness $(h)$ & 2 \\
\hline
\end{tabular}

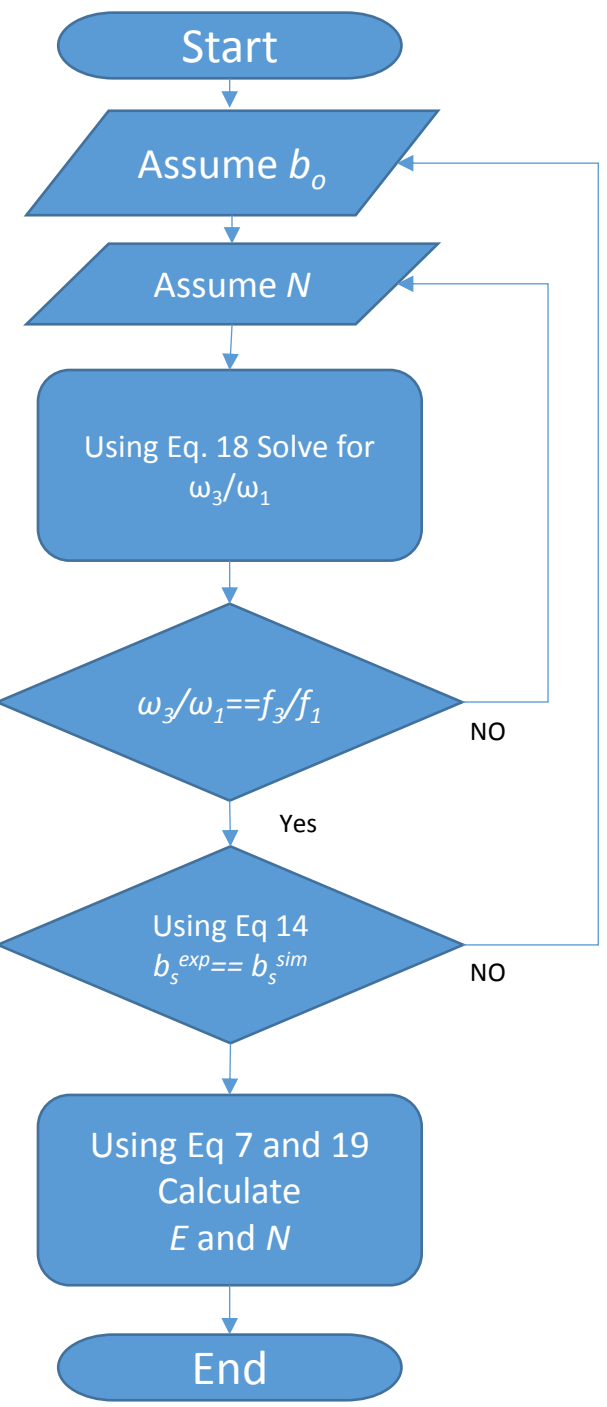

Fig.4. Algorithm steps for finding the induced axial force $N$, the Young's modulus $E$, and the initial rise $b_{o}$.

\section{RESULTS}

\section{A. Static Response}

The static deflection of the arch is investigated using the ZYGO profilometer by forward and backward sweeping the DC voltage load. After applying the voltage load, an image is taken showing the position of the arch with respect to the actuating electrode. Figure 5 shows the experimental setup. Figure 6 shows the DC sweep measurement of the arch. It should be noted that this method of measurement is not so accurate since the resolution of the measurements are within 0.5 micron range. Hence, the static data were not used for the parameter extraction of the device. Also, one can note from Fig. 6 that this arch does not show clear hysteresis, and hence may not have the classical two distinct potential wells, i.e., might be too shallow to have two separate wells. 


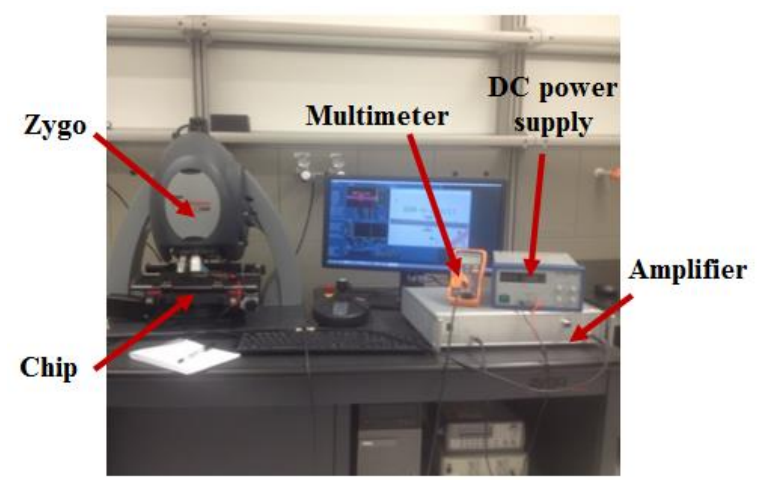

Fig.5. Experimental setup for the static measurement.

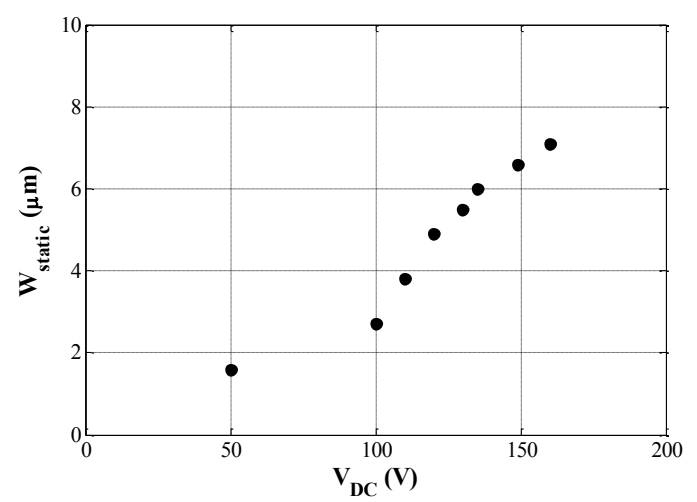

Fig.6. The measured static response.

\section{B. Resonant Frequencies}

In this section, we measure the linear resonance frequencies of the studied arch. We measure the arch resonant frequencies using the Polytec [36] Planar Motion Analyzer (PMA) through applying a ring down square wave signal and then taking the FFT of the measured response. A schematic of the used signal is shown in Fig.7 with its specifications given in Table II.

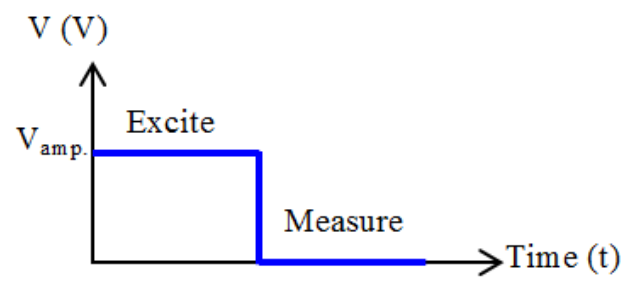

Fig.7. The ring-down signal used to get the resonant frequencies.

TABLE II. The parameters for the ring down measurements.

\begin{tabular}{ll}
\hline Parameter & Value \\
\hline Amplitude & $120 \mathrm{~V}$ \\
Duty cycle & $50 \%$ \\
Frequency limit & $400 \mathrm{kHz}$ \\
Frequency increment & $100 \mathrm{~Hz}$ \\
\hline
\end{tabular}

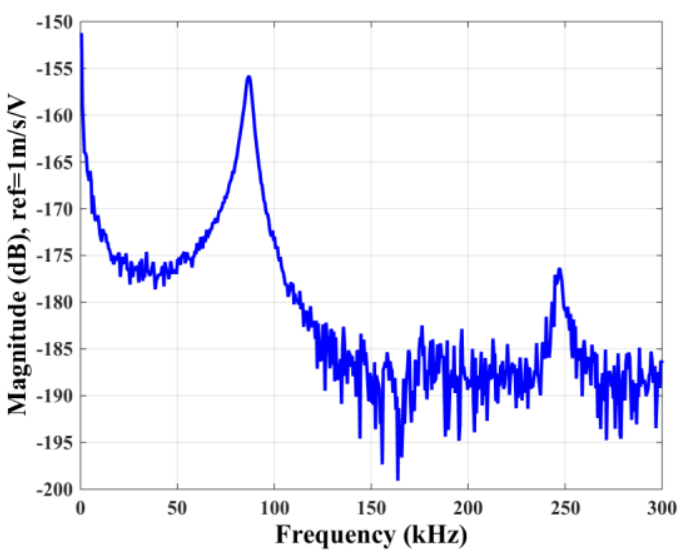

Fig.8. The FFT of the arch in Table I.

Fig. 8 shows the FFT of the resonant frequencies. The first resonance frequency is found at $86.6 \mathrm{kHz}$ and the third resonance frequency (second symmetric) is at $247 \mathrm{kHz}$. Also, one can evaluate the quality factor for each mode from

$$
Q=\frac{f_{\text {resonance }}}{\Delta f}=\frac{f_{\text {resonance }}}{-3 d B_{-} o f_{-} f_{\text {resonance }}}
$$

For the first resonance mode, $Q=19.74$, resulting in a damping ratio $\xi=1 / 2 Q=0.0253$ and for the third resonance frequency, the damping ratio is 0.11 . Hence, these arches are underdamped, which allows for direct harmonic excitations in air. These values will be adopted for all the frequency response curve simulations in Section F.

\section{Excitation around the Primary Resonance Frequency}

Frequency sweep measurements are conducted around the first resonance frequency of the arch. The steady state response at the middle of the arch is measured optically by synchronizing the frequency of the measuring light with the frequency of the device based the on stroboscopic principle. Fig. 9 shows the amplitude of vibration (half of the peak-peak amplitude) of the middle of the arch to forward frequency sweeps for different voltage loads.

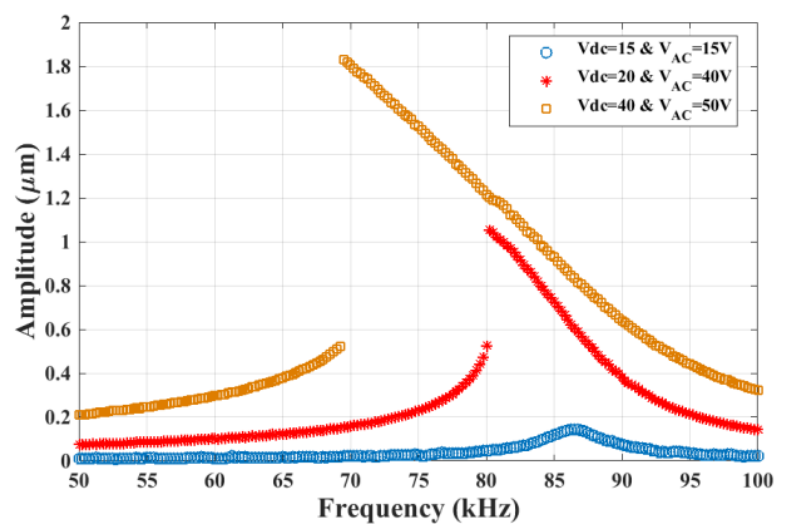

Fig.9. Experimental forward sweeps for various voltage loads around the first resonance frequency of the arch.

From Fig.9, we notice that the dynmic behavior is almost linear at $V_{\mathrm{DC}}=15 \mathrm{~V}$ and $V_{\mathrm{AC}}=15 \mathrm{~V}$. Increasing the voltage 
loads amplify the effect of the quadratic nonlinearity, and hence the stiffness of the arch decreases as the frequency increases. This leads to a softening behavior. Increasing the voltage load shifts the resonant frequency towards lower value. We can notice here the dominance of the quadratic nonlinearity over the cubic nonlinearity in this regime.

\section{Excitation around the Third Resonant Frequency}

The dynamic response around the third mode is also investigated. Figure 10 shows several forward frequency sweeps for different voltage loads. We observe from Fig. 10 that exciting the third mode at a relatively low voltage loads yields an almost linear behavior. By increasing the voltage load, a hardening behavior and jumps are noticed. We can see the dominance of the cubic nonlinearity over the quadratic nonlinearity in this regime.

\section{E. Dynamic Snap-through}

Dynamic snap-through motion is also observed at higher voltage loads near the primary resonance. Figure 11 shows the dynamic snap-through behavior for different voltage loads. In the appendix, we explain this snap-through motion. We notice hardening behavior during the snap-through motion. In addition, increasing the voltage load increases the amplitude of the oscillation. This amplifies the effect of the cubic nonlinearity and hence leads to more stretching. As a result, an increase in the snap-through frequency band is observed. Also, the increase in the voltage load increases the shift in the resonance frequency as noticed.

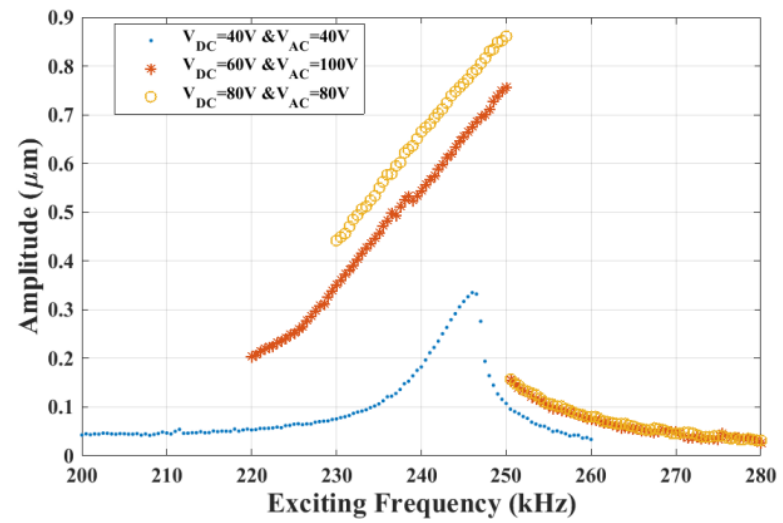

Fig.10. Experimental forward sweeps for various voltage loads around the third mode of the arch.

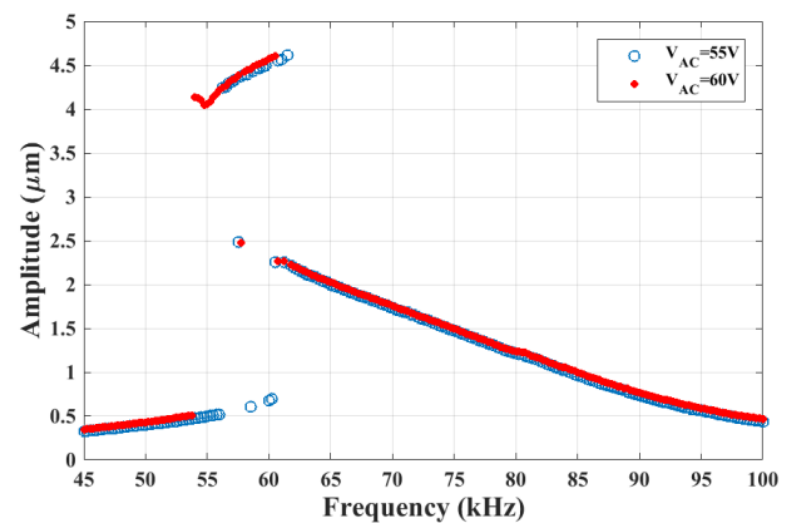

Fig.11. Experimental dynamic snap-though motions at $V_{D C}=$ $60 \mathrm{~V}$ and at different AC voltage loads.

\section{F. Comparison between the Experimental Data and the Theoretical Model Results}

Here, we compare the dynamic response obtained numerically by the reduced order model of Section II and the data obtained experimentally. The first comparison is done at low values of the voltage load as shown in Fig.12. One can see that the response is almost linear. The agreement in this range between the simulation and experiment is excellent.

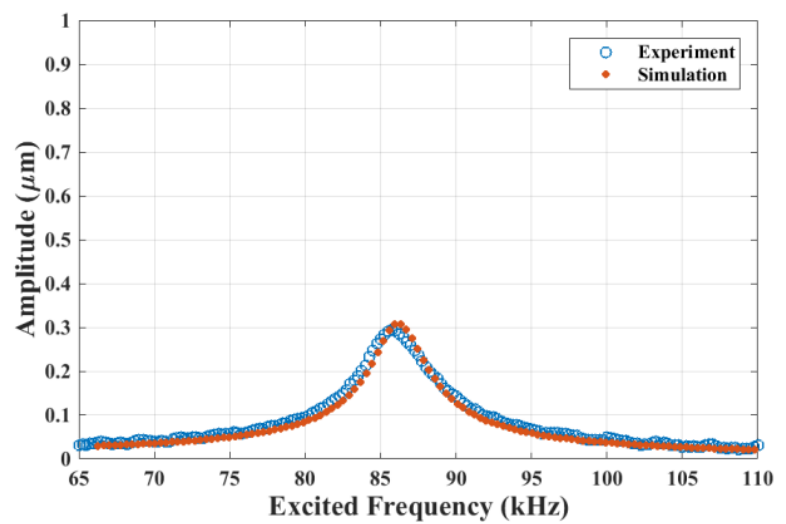

Fig.12. Theoretical and experimental dynamic responses at voltage loads $V_{D C}=15 \mathrm{~V}$ and $V_{A C}=15 \mathrm{~V}$.

Figures 13 and 14 show the comparison for the cases of higher voltage load and the softening behavior effect. Also the agreement here is very good.

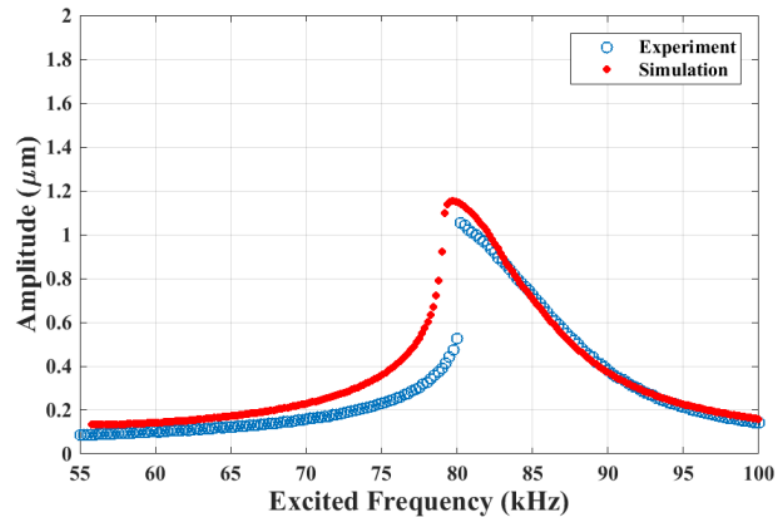

Fig.13. Theoretical and experimental dynamic responses at voltage loads $V_{D C}=20 \mathrm{~V}$ and $V_{A C}=40 \mathrm{~V}$.

Increasing the voltage load gives the system the required energy to jump leading to the dynamic snap-through motion. Figures 15 and 16 compare the numerical simulation and the experimental data in this case of dynamic snap-through motion. As noted, the model in this case predicts the behavior qualitatively and quantitatively with slight discrepancies. This can be attributed to several factors including the nonlinear damping in the case of snap-through motion (since the arch gets very close to the stationary electrodes surrounding it giving rise to squeeze-film like effect) and the non-ideal and flexible supports [28] (since it is observed optically that the 
thickness of the arch near the anchor seems to be thinner than away from the anchors).

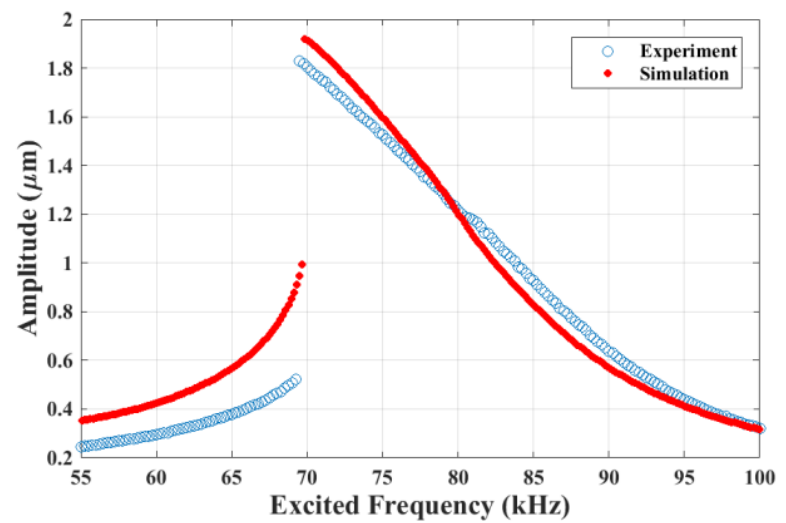

Fig.14. Theoretical and experimental dynamic responses at voltage loads $V_{D C}=40 \mathrm{~V}$ and $V_{A C}=50 \mathrm{~V}$.

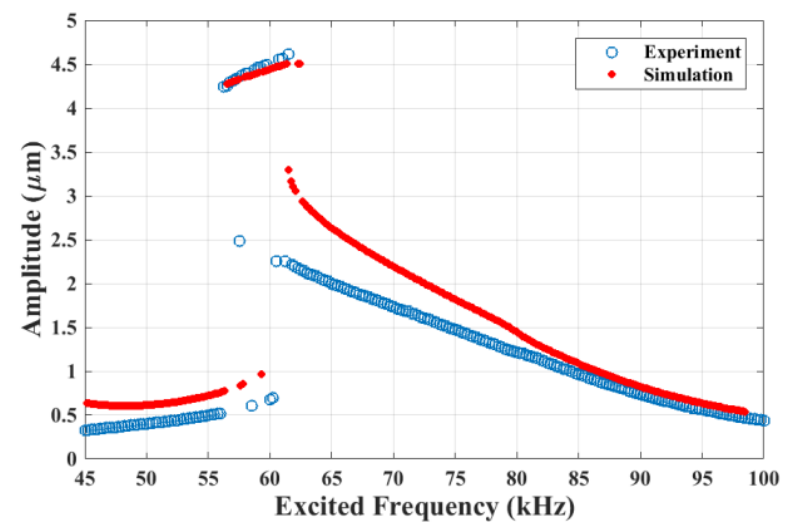

Fig.15. Theoretical and experimental dynamic responses at voltage loads $V_{D C}=60 \mathrm{~V}$ and $V_{A C}=55 \mathrm{~V}$.

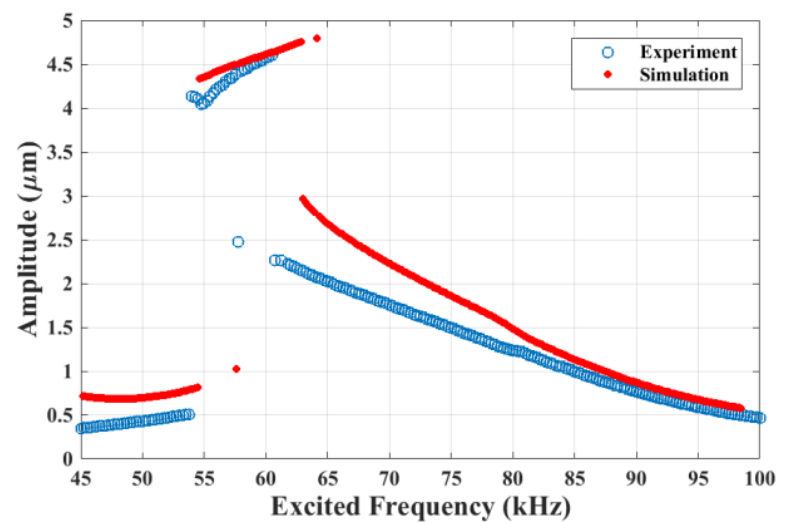

Fig.16. Theoretical and experimental dynamic responses at voltage loads $V_{D C}=60 \mathrm{~V}$ and $V_{A C}=60 \mathrm{~V}$.

\section{CONCLUSIONS}

In this paper, we presented a theoretical and experimental study to investigate the dynamic behavior of an electrostatically actuated in-plane MEMS arch when excited by a DC voltage load superimposed to an AC harmonic voltage load. We developed an algorithm to estimate several unknown parameters of the arch including the internal induced axial force, the initial mid-span rise, and the modulus of elasticity. This algorithm utilizes the experimentally measured modal frequencies and the static rise of the arch after the etching process. Then, we conducted frequency sweeps, revealing softening behaviors occurred for the in-well vibrations. Increasing the voltage load gives the system enough energy to snap-through. A hardening behavior occurred for the dynamic snap-through vibrations and for the second symmetric resonance frequency. Comparison among simulations and experiments revealed good agreement. Our model predicted the behavior qualitatively and quantitatively with slight discrepancies.

\section{APPENDIX}

Using one mode in the reduced-order model, we plot the potential energy of the studied arch. The result is depicted in Fig. 17 at an assumed DC voltage load of $40 \mathrm{~V}$. One can see that this figure reveals a single well potential that changes shape (width) suddenly once the energy exceed certain threshold. This corresponds to the apparent snap-through motion measured dynamically in Fig. 11. Hence, snap-through motion here does not referee to a classic cross-well (between two wells) motion, but rather to a motion due to the sudden expansion of the single well.

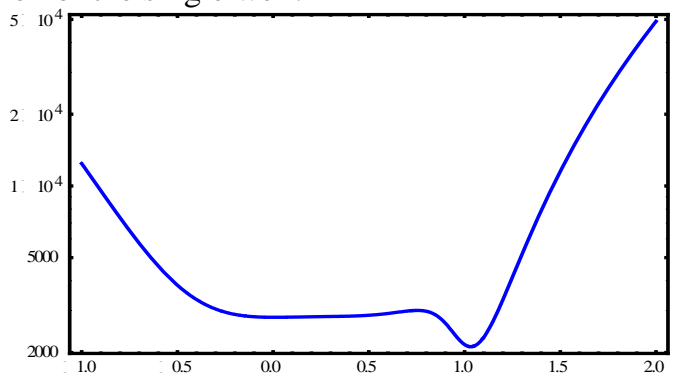

Fig.17. The potential energy of the studied arch under $V_{D C}=$ $40 \mathrm{~V}$.

\section{References}

[1] A. Cabal and D. S. Ross, "Snap-through bilayer microbeam," Technical Proceedings of the 2002 International Conference on Modeling and Simulation of Microsystems, Nanotech 2002, vol. 1, pp. 230-233, 2002.

[2] K. Das and R. C. Batra, "Pull-in and snap-through instabilities in transient deformations of microelectromechanical systems," $J$. of Micromechanics and Microengineering, vol. 19, no. 3, 2009.

[3] Y. Zhang, Y. Wang, and Z. Li, "Analytical method of predicating the instabilities of a micro arch-shaped beam under electrostatic loading," Microsystem Technologies, pp. 909-918, 2010.

[4] S. Krylov, B. R. Ilic, D. Schreiber, S. Seretensky, and H. Craighead," The pull-in behavior of electrostatically actuated bistable microstructures," J. of Micromechanics and Microengineering, vol. 18, no. $5,2008$.

[5] J. Casals-Terre, A. Fargas-Marques, and A. M. Shkel,"Snap-action bistable micromechanisms actuated by nonlinear resonance," J. of Microelectromechanical Systems, vol. 17, no. 5, pp. 1082-1093, 2008.

[6] J. S. Ko, G. L. Min, S. H. Jeong, S. G. Jeung, S. Bosung, and L. DaeSik,"A laterally-driven bistable electromagnetic microrelay," ETRI journal 28, vol. 28, no. 3, pp. 389-392, 2006.

[7] A. Michael and Y. K. Chee, "Design criteria for bi-stable behavior in a buckled multi-layered MEMS bridge," J. of Micromechanics and microengineering, vol. 16, no. 10, pp. 2034, 2006.

[8] X. Song and L. Shi Rong, "Nonlinear stability of fixed-fixed FGM arches subjected to mechanical and thermal loads," Advanced Materials Research, pp. 699-706, 2008.

[9] S. Park, and H. Dooyoung,"Pre-shaped buckled-beam actuators: theory and experiments," Sensors and Actuators A: Physical, vol. 148, no. 1, pp. 186-192, 2008.

[10] S. Krylov, S. Seretensky, and D. Schreiber,"Pull-in behavior and multistability of a curved microbeam actuated by a distributed 
electrostatic force." In Micro Electro Mechanical Systems, 2008. MEMS 2008. IEEE 21st International Conference on, pp. 499-502. IEEE, 2008.

[11] B. Wagner, H. J. Quenzer, S. Hoerschelmann, T. Lisec, and M. Juerss, "Bistable microvalve with pneumatically coupled membranes," In Micro Electro Mechanical Systems. An Investigation of Micro Structures, Sensors, Actuators, Machines and Systems, pp. 384-388, 1996

[12] J. Qiu, J. H. Lang, A. H. Slocum, and A. C. Weber, "A bulkmicromachined bistable relay with U-shaped thermal actuators," J. of Microelectromechanical Systems, vol. 14, no. 5, pp. 1099-1109, 2005.

[13] B. Charlot, W. Sun, K. Yamashita, H. Fujita, and H. Toshiyoshi,"Bistable nanowire for micromechanical memory," $J$. of Micromechanics and Microengineering, vol. 18, no. 4, 2008.

[14] J. Rossiter, B. Stoimenov, and T. A. Mukai,"Self-switching bistable artificial muscle actuator," In SICE-ICASE, (2006). International Joint Conference (pp. 5847-5852). IEEE.

[15] J. S. Han, S. K. Jong, T. K. Youn, and M. K. Byung, "Parametric study and optimization of a micro-optical switch with a laterally driven electromagnetic microactuator," J. of Micromechanics and Microengineering, vol. 12, no. 6, pp. 939, 2006.

[16] M. I. Younis, "MEMS Linear and Nonlinear Statics and Dynamics": MEMS Linear and Nonlinear Statics and Dynamics. Springer Science \& Business Media, (2011).

[17] G. Sari and M. Pakdemirli, "Vibrations of a Slightly Curved Microbeam Resting on an Elastic Foundation with Nonideal Boundary Conditions," Mathematical Problems in Engineering, vol. 2013, 2013.

[18] R. H. Plaut,"Snap-through of arches and buckled beams under unilateral displacement control," Int. J. of Solids and Structures, vol. 63, pp. 109$113,2015$.

[19] N. Hu and R. Burgueño, "Buckling-induced smart applications: recent advances and trends," Smart Materials and Structures, vol. 24, no. 6, 2015.

[20] A. H. Nayfeh, W. Kreider, and T. J. Anderson,"Investigation of natural frequencies and mode shapes of buckled beams," AIAA J., vol. 33, no. 6, pp. 1121-1126, 1995.

[21] D. J. Dawe, "The transverse vibration of shallow arches using the displacement method," Int. J. of Mechanical Sciences, vol. 13, no. 8, pp. 713-720, 1971.

[22] H. M. Ouakad and M. I. Younis, "The dynamic behavior of MEMS arch resonators actuated electrically," Int. J. of Non-Linear Mechanics, vol. 45, no. 7, pp. 704-713, 2010.

[23] S. A. Alkharabsheh, and M. I. Younis, "Statics and dynamics of MEMS arches under axial forces," J. of Vibration and Acoustics, vol. 135, 2013.

[24] N. J. Mallona, R. H. B. Fey, H. Nijmeijer, and G. Q. Zhang, "Dynamic buckling of a shallow arch under shock loading considering the effects of the arch shape," Int. J. of Non-Linear Mechanics, vol. 41, no. 9, pp. 1057-1067, 2006.

[25] J. Lv, Y. Zhuangpeng, and W. Ronghui, "Dynamic Stability of Shallow Arches with the Geometrical Imperfections," Computational Sciences and Optimization, vol. 2, pp. 691-695, 2009.

[26] L. Ruzziconi, A. M. Bataineh, M. I. Younis, W. Cui, and S. Lenci, "Nonlinear dynamics of an electrically actuated imperfect microbeam resonator: experimental investigation and reduced-order modeling," J. of Micromechanics and Microengineering, vol. 23, no. 7, 2013.

[27] L. Buchaillot, O. Millet, E. Quévy, and D. Collard, "Post-buckling dynamic behavior of self-assembled 3D microstructures," Microsystem Technologies, vol. 14, no. 1, pp. 69-78, 2008.

[28] S. A. Alkharabsheh and M. I. Younis,"Dynamics of MEMS arches of flexible supports," J. of Microelectromechanical Systems, vol. 22, no. 1, pp. 216-224, 2013.

[29] L. Medina, R. Gilat, B. Ilic, and S. Krylov, "Experimental investigation of the snap-through buckling of electrostatically actuated initially curved pre-stressed micro beams," Sensors and Actuators, vol. 220, pp. 323332, 2014.

[30] M. I. Younis, H. M. Ouakad, F. M. Alsaleem, R. Miles, and W. Cui, "Nonlinear dynamics of MEMS arches under harmonic electrostatic actuation," J. of Microelectromechanical Systems, vol. 19, no. 3, pp. 647-656, 2010

[31] M. I. Younis, E. M. Abdel-Rahman, and A. H. Nayfeh, "A reducedorder model for electrically actuated microbeam-based MEMS," J. of Microelectromechanical Systems, vol. 12, no. 5, pp. 672-680, 2013.

[32] L. Medina, R. Gilat, B. Ilic, and S. Krylov, "Dynamic trapping experiment in an elctrostaticlly actuated initially curved beam" IEEE 18th International Conference on Solid-State Sensors, Actuators and
Microsystems (TRANSDUCERS), 2015 Transducers, Ak. pp. 784 - 787 doi: 10.1109/TRANSDUCERS.2015.7181040.

[33] A. H. Nayfeh, M. I. Younis, and E. M. Abdel-Rahman,"Reduced-order models for MEMS applications," Nonlinear Dynamics, vol. 41, no. 1, pp.211-236, 2005.

[34] MEMSCAP. http://www.memscap.com/

[35] M. A. Hopcroft, W. D. Nix and T. W. Kenny, "What is the Young's Modulus of Silicon?," Journal of Microelectromechanical Systems, 19, 2, pp. 229-238, 2010.

[36] Polytec. http://www.polytec.com/us/ 